\title{
Dalfampridine sustained-release for symptomatic improvement of walking speed in patients with multiple sclerosis
}

This article was published in the following Dove Press journal:

Core Evidence

9 December 2010

Number of times this article has been viewed

\author{
Douglas R Jeffery' \\ Emily Poole Pharr ${ }^{2}$ \\ 'MS Center at Advance Neurology, \\ Advance, NC, USA; ${ }^{2}$ Department of \\ Neurology, Wake Forest University \\ Health Science, Winston-Salem, \\ NC, USA
}

\begin{abstract}
Dalfampridine sustained-release (SR) is a time-release formulation of 4-aminopyridine, recently approved by the Food and Drug Administration to improve walking in patients with multiple sclerosis (MS). In Phase II trials, walking speed and lower extremity muscle strength was increased in patients with MS, but the increase in walking speed did not reach statistical significance. A responder analysis revealed that approximately $35 \%$ of treated patients had a statistically significant and clinically meaningful increase in walking speed. When treated responders were compared with treated nonresponders, walking speed significantly increased in the responder group, but not in the nonresponder or placebo groups. This result was duplicated in two larger Phase III trials. The optimal dose to maximize the risk-benefit ratio was $10 \mathrm{mg}$ twice daily. Higher doses were associated with a greater risk of seizure, but no further improvement in walking speed or in the proportion of responders Dalfampridine SR is eliminated by renal clearance and undergoes only limited metabolism $(<10 \%)$. It is contraindicated in patients with moderate or severe renal insufficiency and in those with a history of seizures or epileptiform activity on electroencephalography. The development of time-released 4-aminopyridine represents a major advance in symptomatic therapy for MS.
\end{abstract}

Keywords: dalfampridine SR, multiple sclerosis, ambulation, walking speed, clinical trials, safety

Core evidence clinical impact summary for dalfampridine sustained-release in multiple sclerosis

\begin{tabular}{|c|c|c|}
\hline Outcome measure & Evidence & Implications \\
\hline \multirow[t]{3}{*}{$\begin{array}{l}\text { Disease-oriented } \\
\text { evidence }\end{array}$} & Phase II study & $\begin{array}{l}\text { First drug approved for the } \\
\text { symptomatic } \\
\text { management of multiple sclerosis }\end{array}$ \\
\hline & Phase III studies & $\begin{array}{l}\text { Broad indication to improve walking in } \\
\text { patients with MS }\end{array}$ \\
\hline & $\begin{array}{l}\text { Small clinical trials with } \\
\text { 4-aminopyridine }\end{array}$ & $\begin{array}{l}\text { This agent will be used widely and } \\
\text { may be } \\
\text { of substantial benefit to patients with } \\
\text { MS }\end{array}$ \\
\hline $\begin{array}{l}\text { Patient-oriented } \\
\text { evidence }\end{array}$ & $\begin{array}{l}\text { Responders showed } \\
\text { improvements in the MS Walking } \\
\text { Scale and Subject Global Index } \\
\text { and Clinician Global Index }\end{array}$ & $\begin{array}{l}\text { Quality of life may be improved in } \\
\text { responders }\end{array}$ \\
\hline Economic evidence & None & \\
\hline
\end{tabular}

Correspondence: Douglas R Jeffery Director of the MS Center at Advance Neurology, 152 Kinderton Way,

Suite I0I, Advance,

NC 27006, USA

$\mathrm{Tel}+\mathrm{I} 336940278$ I

Fax +I 3369402782

Email douglas.jeffery@

cornerstonehealthcare.com submit your manuscript | www.dovepress.com

Dovepress

DOI: $10.2147 /$ CE.S9046 


\section{Introduction}

Multiple sclerosis (MS) affects over 400,000 people in the US and over a million people globally. ${ }^{1}$ In the last 15 years, significant advances have been made in the pharmacologic treatment of the inflammatory disease activity that partly defines the disease, but there has been little progress in the development of symptomatic therapies. This has been a significant unmet need in the treatment of MS.

Patients suffering from MS experience a variety of symptoms that negatively impact their quality of life. These include weakness, sensory loss, impaired coordination, cognitive dysfunction, pain, malaise, and bladder dysfunction, to name only a few. ${ }^{2}$ One of the challenges faced by clinicians treating MS is the amelioration of what its symptoms cause. This is often more challenging than bringing inflammatory disease activity under control. In one study, $64 \%$ of MS patients reported difficulty with walking, and in that group 78\% reported that difficulty walking impaired their ability to work and $82 \%$ reported that it restricted their activities. ${ }^{3}$

In addition to the aforementioned symptoms, many patients have fatigability of strength. This is defined as a significant decrease in strength that occurs with repetitive movements. For example, if one lifts a $10 \mathrm{~kg}$ weight many times, the muscle will fatigue and the movement will become impossible to repeat. This phenomenon occurs normally, but in patients with MS it occurs much earlier, and is due to lesions within the central nervous system (CNS) that have not completely interrupted synaptic transmission. When high volumes of nerve impulses travel through damaged regions, action potentials slow and there is a dispersion of action potential volleys, leading to a decreased response. ${ }^{4}$ This is manifested as weakness when a motor pathway has been affected. This occurs quite commonly in patients with MS, so that even though ambulation may be initially normal, after the patient has walked a given distance, leg strength decreases and the patient must stop and rest. As a result, the development of an agent to improve walking in patients with MS has been a significant unmet need in MS therapeutics.

\section{Development of dalfampridine}

4-Aminopyridine (4-AP) is a voltage-gated potassium channel blocker that results in an increase in the duration of the action potential. Potassium efflux out of the axon is the ionic mechanism that results in repolarization of the axon. This must occur before the axon can propagate another action potential. In MS, myelinated fibers are damaged, and when remyelination does occur, the sheath is thinner than those seen in undamaged fibers. The result is that there is an impedance mismatch and dispersal of action potentials within a volley traveling down the axon. This results in either a weakened signal when the volley of action potentials reaches its destination or in outright conduction block. Clinically, this is manifested as weakness and in fatigability of strength. 4-AP increases the conduction of action potentials in demyelinated fibers, and in doing so, may result in improvement of strength. The administration of 4-AP may also result in increased neuronal excitability and in the potentiation of synaptic transmission. ${ }^{5}$ The latter may be due to increased release of neurotransmitter that occurs as a result of increased calcium influx at presynaptic terminals. Its action and toxicity could be due to either mechanism. As a result, 4-AP may lower the seizure threshold, and seizures have occurred in patients on compounded 4-AP.

Studies of 4-AP in a compounded formulation date back many years and have demonstrated significant benefits in MS patients. Several trials have demonstrated that some, but not all, patients experience improvement in motor function, ambulatory speed, and visual function. ${ }^{6-9}$ Others have demonstrated an improvement in the fatigue severity scale and a decrease in the Expanded Disability Status Scale (EDSS). ${ }^{9}$ As a result of the positive results reported in these studies, 4-AP has been widely used in clinical practice. Unfortunately, the reliability of the preparations produced in compounding pharmacies has been poor. As a result, the actual amount of 4-AP in compounded product has varied widely, and this has led to unintentional overdose in some cases and inadequate amounts in other preparations. ${ }^{10,11}$ Further, serum levels of 4-AP following administration of immediate-release 4-AP rise quite rapidly and peak at 1.2 hours. ${ }^{12}$ The serum level rapidly declines and this necessitates frequent dosing. The therapeutic index is quite narrow, which can lead to levels that are either too high or too low. Peak serum concentrations following immediate-release 4-AP are high enough to increase the risk of seizure significantly.

A time-release formulation of 4-AP (dalfampridine) has been developed by Acorda Therapeutics and has recently been approved by the Food and Drug Administration (FDA) to improve walking in patients with MS. This review will summarize the data demonstrating the safety and efficacy of dalfampridine and discuss the scope of its use in the MS population.

\section{Pharmacokinetics}

Dalfampridine is highly lipid-soluble and readily penetrates the CNS, blocking potassium channels centrally and in the peripheral nervous system. Maximal serum concentration is 
reached approximately three hours following oral administration and the peak serum concentration is approximately $20 \mathrm{ng} / \mathrm{mL}$. Peak serum concentrations occur at 1.2 hours after administration of an immediate-release formulation and reach levels of $46 \mathrm{ng} / \mathrm{mL} .{ }^{12}$ The half-life of dalfampridine sustained-release (SR) is 6.4 hours versus 3.7 hours for an immediate-release preparation. Twelve hours after dosing, the serum concentration of dalfampridine SR is approximately $12 \mathrm{ng} / \mathrm{mL}$. The drug is almost completely eliminated within 24 hours. ${ }^{13-15}$ Bioavailability is $96 \%$, and there is very little binding to plasma proteins $(1 \%-3 \%)$. There is minimal metabolic transformation. Ninety percent of an administered dose is excreted in the urine as the parent compound. A small amount is metabolized by CYP2E1, which results in two metabolites, neither of which has activity on potassium channels. In patients with renal insufficiency, peak dalfampridine SR concentrations are increased. In patients with mild renal impairment, as defined by a creatinine clearance of 50-80 $\mathrm{mL} / \mathrm{min}$, peak concentrations were increased by $166 \%-200 \%$. In those with severe impairment, as defined by a creatinine clearance of less than $30 \mathrm{~mL} / \mathrm{min}$, peak serum concentrations were increased by $175 \%-398 \%{ }^{13}$ Terminal half-life was also increased in patients with renal impairment. In those with mild, moderate, or severe impairment, half-life was 7.4 hours, 8.1 hours, and 14.3 hours, respectively. Therefore, its use in patients with moderate or severe renal impairment is contraindicated. In patients with mild renal insufficiency, serum concentrations could be increased and there is greater potential for adverse events. When the drug is taken with food, there is a modest $(12 \%-17 \%)$ increase in peak serum concentration. Therefore, the drug may be taken with or without food.

\section{Clinical trials}

An early Phase II trial of dalfampridine SR in MS enrolled 10 patients who were clinically stable, with motor deficits and an EDSS of 6.0 and 7.5. ${ }^{7}$ Patients were allowed to remain on other symptomatic medications and were randomized to receive dalfampridine SR 17.5 twice daily or placebo. Following the treatment period, patients were washed out for one week and then crossed over, so that the patients previously receiving dalfampridine SR were switched to placebo and vice versa. A number of outcome measures were used, including EDSS, grip strength, global impression, time to walk $8 \mathrm{~m}$, and manual muscle strength testing. The only outcome measure that reached statistical significance was the timed walk. On that measure, nine of 10 patients had an increased walking speed $(P=0.02)$. Seven of 10 patients reported improved global impression scores on dalfampridine SR compared with placebo. There were no serious adverse events and no patient had a seizure. Minor adverse events included dizziness, insomnia, and paresthesias.

Of note is that subjective improvement and improvement on some outcome measures was related to the serum level. In patients whose serum level exceeded $60 \mathrm{ng} / \mathrm{mL}$, there was improvement in timed walking, grip, and manual muscle strength testing. In addition, only patients whose serum concentrations exceeded $60 \mathrm{ng} / \mathrm{mL}$ reported subjective improvement.

Next came a further Phase II, double-blind, placebocontrolled trial to determine the safety and efficacy of three doses of dalfampridine SR. ${ }^{16}$ In this trial, 206 patients were randomized to treatment with dalfampridine SR at doses of $10 \mathrm{mg}, 15 \mathrm{mg}$, or $20 \mathrm{mg}$ twice daily. Patients had to have a timed 25-foot walk (T25FW) of 8-60 seconds and be aged $18-70$ years. The primary outcome measure was change in T25WF, and secondary outcome measures included the lower extremity manual muscle strength test, Ashworth scale, MS functional composite, and others. The trial utilized a twoweek run-in phase for dose escalation in the higher dose groups. Assessments were carried out at baseline, during the 12-week stable dose phase, and after cessation of dosing.

The trial failed to demonstrate a statistically significant effect on the primary outcome measure, even though walking speed was faster for each of the treated groups compared with the placebo-treated group. There were improvements in lower extremity muscle strength, $P=0.018$ for the $10 \mathrm{mg}$ group and $P=0.003$ for the $15 \mathrm{mg}$ group. There were no statistically significant differences between groups for any of the other secondary outcome measures.

Following these analyses, a post hoc responder analysis was carried out. The mean increase in walking speed in the treated groups suggested that some, but not all, patients may have responded to treatment. The responder analysis was based on the criteria that a responder would have an improvement in walking speed while on drug that would reverse off drug. This was operationally defined as a patient whose walking speed on at least three visits during the double-blind treatment period was faster than the maximum speed achieved in the five off-treatment visits.

Using this criterion, the response rate was $8.5 \%$ in the placebo group, $35.3 \%$ in the $10 \mathrm{mg}$ group, $36 \%$ in the $15 \mathrm{mg}$ group, and $38.6 \%$ in the $20 \mathrm{mg}$ group. These differences all achieved statistical significance. In the dalfampridine SR treated responder groups, walking speed increased from $25 \%$ to $29 \%$. This degree of improvement is considered clinically 
significant. ${ }^{17,18}$ Also of note is that the change in walking speed in the responder groups treated with dalfampridine SR was maintained over the 12 -week treatment period. In the responder groups, there were statistically significant improvements in the MS Walking Scale $(P=0.020)$ and on the Subject Global Index $(P=0.004)$. Unfortunately, there was no association between responder status and any demographic variable. Neither age, EDSS, MS subtype, nor any other variable predicted responder status.

Dalfampridine SR was generally well tolerated. There was no difference in the overall frequency of adverse events between the $10 \mathrm{mg}$ dose group and placebo. In general, there was an increased risk of serious adverse events in the $15 \mathrm{mg}$ and $20 \mathrm{mg}$ dose groups. In the $15 \mathrm{mg}$ dose group, $8 \%$ experienced serious adverse events and, in the $20 \mathrm{mg}$ dose group, $12 \%$ experienced serious adverse events. In the $15 \mathrm{mg}$ group, one patient discontinued treatment due to nausea and dizziness. In the $20 \mathrm{mg}$ dose group, five patients discontinued treatment. Two of those patients experienced seizures, one had abnormal coordination, one had chest pain and headache, and one other had blurred vision, balance disorder, headache, and paresthesia.

The results of this study suggested that a subset of MS patients with motor dysfunction and impaired walking responded to dalfampridine SR. Importantly, another group indistinguishable on the basis of clinical characteristics did not respond. Overall, safety and tolerability appeared quite good in the $10 \mathrm{mg}$ dose group and the clinical benefits in the responder group were enhanced by the use of higher doses which posed a greater risk of adverse events. Therefore, further Phase III trials used dalfampridine SR at $10 \mathrm{mg}$ twice daily and included a prospective responder analysis.

The next trial to determine efficacy was a randomized, multicenter, double-blind, Phase III trial in 296 patients with MS. ${ }^{17}$ Inclusion criteria included being aged $18-70$ years, with any MS subtype, and a T25FW of 8-45 seconds at screening. Those with a history of seizures or epileptiform activity on a screening electroencephalogram (EEG) were excluded from the study. Patients meeting the inclusion criteria were randomized to placebo or dalfampridine SR $10 \mathrm{mg}$ twice daily. The primary outcome measure was response to treatment, using the T25TW as the determinant of response. Secondary outcome measures included the Ashworth scale and lower extremity manual muscle strength test. The MS Walking Scale, Subject Global Impression, and Clinician Global Impression were also employed as tertiary outcome measures.

Using the responder analysis, there were significantly more responders in the dalfampridine SR group than in the placebo group (35\% versus $8 \% ; P<0.0001)$. The average change from baseline in walking speed in the responder group was $25.2 \%$. This increase was maintained over the 14 -week treatment period and, when the drug was discontinued, walking speed returned to rates observed at screening, suggesting that the responder analysis accurately captured those who demonstrated a beneficial effect. Effects on secondary outcome measures also reached statistical significance. Responders had decreased scores on the MS Walking Scale (-6.84) compared with nonresponders $(0.05 ; P=0.0002$. Significant differences were reported favoring dalfampridine SR responders on the Subject Global Impression, Clinician Global Impression, and lower extremity muscle strength test as well.

Safety and tolerability in this Phase III trial was consistent with the results of the Phase II study showing good tolerability and an acceptable safety profile. Eleven (5\%) of patients in the dalfampridine SR group discontinued the study due to adverse events. Two serious adverse events in the dalfampridine SR group were considered to be possibly drug-related. The first was a patient with worsening anxiety on treatment. When treatment was withheld, this resolved but reappeared when treatment was started again. The second patient experienced a seizure in the context of pneumonia and a urinary tract infection. Milder adverse events more common in the dalfampridine SR group were also consistent with adverse events seen in the Phase II trial, and included dizziness, nausea, insomnia, fatigue, and impaired balance.

The results of this trial confirm those of the Phase II study and suggest that roughly $35 \%$ of MS patients with fixed motor weakness and gait impairment may respond to and benefit from dalfampridine SR. No unexpected adverse events occurred during the course of the trial, suggesting an acceptable risk-benefit ratio.

A second Phase III trial intended to provide supporting data for registration has been completed. ${ }^{19}$ This trial enrolled 239 patients with MS. Inclusion criteria were similar to the first Phase III trial. Patients with MS and aged $18-70$ years were enrolled. Patients must have had motor weakness and gait impairment with a T25FW of 8-45 seconds. Patients were excluded if they had a history of seizures or epileptiform activity on EEG. After the screening visit, patients were randomized to placebo or dalfampridine SR, and underwent a two-week run-in phase prior to a nine-week treatment phase. Following the end of the nine-week treatment phase, the drug was discontinued and patients were reassessed two weeks after cessation of treatment.

The primary outcome measure in this trial was the proportion of patients responding to treatment. A treatment 
responder was defined as a patient who had a faster walking speed on three out of four treatment visits than any maximum speed during five off-treatment visits. Off-treatment visits were those at screening, during the run-in period, and after treatment cessation. In this fashion, a fairly stringent criterion for treatment response was defined.

The patient population consisted of all MS subtypes. Approximately 33\% were relapsing-remitting, 8\%-17\% were primary progressive, $47 \%-51 \%$ were secondarily progressive, and 2\%-4\% were progressive relapsing. EDSS scores ranged from 1.5 to 7.0 . Approximately $43 \%$ of the patients treated with dalfampridine SR were classified as responders compared with only $9 \%$ percent of the placebo group. This was similar to the results reported in the earlier Phase III study, and suggested that dalfampridine SR may bring about a clinically significant improvement in walking speed and strength in a substantial portion of the MS patient population. In the dalfampridine $\mathrm{SR}$ responders, walking speed increased by about $25 \%$ compared with placebo and with nonresponders. Of interest, the patients who did not respond had walking speeds quite comparable with those of placebo-treated patients. In addition, walking speed was measured at intervals following the last dose, and this analysis showed that 12 hours after the last dose of dalfampridine SR walking speed decreased, supporting twice-daily dosing. Responders also had significant improvements in MS Walking Scale 12 hours after the last dose of dalfampridine SR.

A pooled analysis of all patients enrolled in the Phase II trial and both Phase III dalfampridine SR trials showed statistically significant improvements in walking speed in an analysis that compared placebo with treated patients and did not distinguish between responders and nonresponders. ${ }^{20}$ The average improvement in walking speed in the pooled data set was $13.4 \%$. In total, $31.5 \%$ showed at least a $20 \%$ improvement in walking speed and $15.5 \%$ showed a $30 \%$ increase in walking speed. Adverse events in the pooled sample population were similar to those reported in earlier studies, and included an increased frequency of asthenia, fatigue, dizziness, headache, nausea, and impaired balance. Of note is that there was also an increased risk of urinary tract infection (14.5\% in dalfampridine SR versus $9.2 \%$ in placebo).

\section{Use of dalfampridine SR in clinical practice}

Dalfampridine SR was approved by the FDA on the basis of the studies reviewed in this paper. It was given a very broad indication, ie, "to improve walking in patients with MS".
The FDA did not restrict its use to those with an EDSS of 2.5-7.0 nor to patients with a T25FW of 8-45 seconds. This is the first agent ever to be approved for the symptomatic management of MS (see Table 1). Its use could benefit 30\%-40\% of the MS population. Unfortunately, there are no predictors of a positive response to dalfampridine SR. Therefore, its benefit or lack thereof will have to be determined empirically in each patient. If patients perceive benefit, they will continue to take the drug and, if not, they will likely discontinue it on their own.

Of note is that dalfampridine SR could be useful to improve other mobility function in MS. It is possible, although there are no data to support it, that patients with limited upper extremity function could benefit. Likewise, it is also possible that patients with limited ability to transfer could benefit from treatment with dalfampridine SR. This will have to be determined in Phase IV studies.

Dalfampridine SR is contraindicated in patients with a history of seizures and may increase the risk of seizures. The increased risk of seizures at $10 \mathrm{mg}$ twice daily was minimal but the possibility remains. It is also contraindicated in patients with moderate and severe renal impairment, and its use in patients with mild renal impairment should be undertaken with caution. Patients must also be warned that taking additional doses of dalfampridine SR will not bring about further benefit and could increase the risk of seizures.

The introduction of dalfampridine SR represents an important improvement in the care of MS patients. It is the first drug approved by the FDA for the symptomatic management of MS patients, and has the capability to improve function and quality of life in those with motor weakness and ambulatory impairment.

\section{Disclosure}

Dr Poole reports no conflicts of interest in this work. Dr Jeffery has received honoraria for speaking and consulting from Bayer, Biogen, Serono, Pfizer, Teva, Acorda, Novartis, and GSK. He has also received research support from Bayer, Biogen, Teva, Serono, Pfizer, and Novartis.

\section{References}

1. Noonan CW, Kathman SJ, White MC. Prevalence estimates for MS in the United States and evidence of an increasing trend for women. Neurology. 2002;58:136-138

2. Noseworthy JH, Lucchinetti C, Rodriguez M, Weinshenker BG. Multiple sclerosis. N Engl J Med. 2000;343:938-952.

3. National Multiple Sclerosis Society. Loss of mobility found to impact quality of live and emotional and financial health of most people living with multiple sclerosis. Available at: http:/www.nationalmssociety.org news/news-detail/index.aspx?nid=199. Accessed 2009 Dec 8. 
4. Herndon RM. Pathology and pathophysiology. In: Burks JS, Johnson KP, editors. Multiple Sclerosis: Diagnosis, Medical Management, and Rehabilitation. New York, NY: Demos Medical Publishing; 2000 .

5. Sherratt RM, Bostock H, Sears TA. Effects of 4-aminopyridine on normal and demyelinated mammalian nerve fibres. Nature. 1980; 283: 570-572.

6. Bever CT, Young D, Anderson PA, et al. The effects of 4-aminopyridine in multiple sclerosis patients: Results of a randomized, placebocontrolled, double-blind, concentration-controlled, crossover trial. Neurology. 1994;44:1054-1059.

7. Schwid SR, Petrie MD, McDermott MP, et al. Quantitative assessment of sustained-release 4-aminopyridine for symptomatic treatment of multiple sclerosis. Neurology. 1997;48:817-821.

8. van Diemen HAM, Polman CH, van Dongen TM, et al. The effect of 4-aminopyridine on clinical signs in multiple sclerosis: A randomized, placebo-controlled, double-blind, cross-over study. Ann Neurol. 1992; 32:123-130

9. Stefoski D, Davis FA, Faut M, Schauf CL. 4-Aminopyridine improves clinical signs in multiple sclerosis. Ann Neurol. 1987;21:71-77.

10. Schwam E. Severe accidental overdose of 4-aminopyridine due to a compounding pharmacy error. Emerg Med. 2009 May 12. [Epub ahead of print].

11. Burton JM, Bell CM, Walker SE, O'Connor PW. 4-aminopyridine toxicity with unintentional overdose in four patients with multiple sclerosis. Neurology. 2008;71:1833-1834.

12. Hayes KC, Katz MA, Devane JG, et al. Pharmacokinetics of an immediaterelease oral formulation of fampridine (4-aminopyridine) in normal subjects and patients with spinal cord injury. J Clin Pharmacol. 2003; 43:379-385.
13. Smith W, Swan S, Marbury T, et al. Single-dose pharmacokinetics of sustained-release fampridine (fampridine-SR) in healthy volunteers and adults with renal impairment. J Clin Pharmacol. 2010;50: 151-159.

14. Vollmer T, Henney HR. Pharmacokinetics and tolerability of single escalating doses of Fampridine sustained-release tablets in patients with multiple sclerosis: A phase I-II, open-label trial. Clin Ther. 2009;31: 2206-2214.

15. Vollmer T, Blight AR, Henney HR. Steady-state pharmacokinetics and tolerability of orally administered fampridine sustained-release 10-mg tablets in patients with multiple sclerosis: A 2-week, open-label, follow-up study. Clin Ther. 2009;31:2215-2223.

16. Goodman AD, Brown TR, Cohen JA, et al. Dose comparison trial of sustained-release fampridine in multiple sclerosis. Neurology. 2008; 71:1134-1141.

17. Goodman AD, Brown TR, Krupp LB, et al. Sustained-release oral fampridine in multiple sclerosis: A randomized, double-blind, controlled trial. Lancet. 2009;373:732-738.

18. Hobart JC, Riazi A, Lamping DL, et al. Measuring the impact of MS on walking ability: The 12-item MS walking scale (MSWS-12). Neurology. 2003;60:31-36.

19. Goodman AD, Schwid SR, Brown TR, et al. Sustained-release fampridine consistently improves walking speed and leg strength in multiple sclerosis: A phase 3 trial. Mult Scler. 2008;14:S295.

20. Schapiro R, Goodman AD, Brown T, Marinucci L, Cohen R, Blight A. Dalfampridine improves walking in MS patients: Pooled data from three clinical trials. 24th Annual Meeting of the Consortium of Multiple Sclerosis Centers. 2010;S114. Available from: http:// mscare.org/cmsc/images/pdf/IJMSC-2010-May-Supp.pdf. Accessed 2010 Sep 30.
Core Evidence

\section{Publish your work in this journal}

Core Evidence is an international, peer-reviewed open-access journal evaluating the evidence underlying the potential place in therapy of drugs throughout their development lifecycle from preclinical to postlaunch. The focus of each review is to evaluate the case for a new drug or class in outcome terms in specific indications and patient groups.

\section{Dovepress}

The manuscript management system is completely online and includes a very quick and fair peer-review system, which is all easy to use. Visit http://www.dovepress.com/testimonials.php to read real quotes from published authors. 